TRANSACTIONS OF THE

AMERICAN MATHEMATICAL SOCIETY

Volume 349, Number 5, May 1997, Pages 1783-1796

S 0002-9947(97)01900-4

\title{
CORRELATION DIMENSION FOR ITERATED FUNCTION SYSTEMS
}

\author{
WAI CHIN, BRIAN HUNT, AND JAMES A. YORKE
}

\begin{abstract}
The correlation dimension of an attractor is a fundamental dynamical invariant that can be computed from a time series. We show that the correlation dimension of the attractor of a class of iterated function systems in $\mathbf{R}^{N}$ is typically uniquely determined by the contraction rates of the maps which make up the system. When the contraction rates are uniform in each direction, our results imply that for a corresponding class of deterministic systems the information dimension of the attractor is typically equal to its Lyapunov dimension, as conjected by Kaplan and Yorke.
\end{abstract}

\section{INTRODUCTION}

The dimension spectrum $D_{q}$ is a group of important invariants characterizing attractors of dynamical systems [G] [HP]. Of particular interest are the box-counting (or capacity) dimension $D_{0}$, the information dimension $D_{1}$, and the correlation dimension $D_{2}$, which are related by the inequalities $D_{0} \geq D_{1} \geq D_{2} . D_{0}$ measures the dimension of the attractor as a set, while $D_{q}$ for $q>0$ depends on the distribution of points induced by the dynamics on the attractor. $D_{1}$ is conjectured for a typical attractor to equal the "Lyapunov dimension", a function of the attractor's Lyapunov exponents; this is referred to as the Kaplan-Yorke conjecture [KY] [FOY]. $D_{2}$ has received much attention recently from dynamicists and is generally the easiest of the dimensions to compute from experimental data [GP] $[\mathrm{P}]$.

The correlation dimension $D_{2}$ also admits a potential-theoretic definition [DGOSY] [SY] which we exploit, along with the above inequalities, to prove that the Kaplan-Yorke conjecture holds for the attractors of a class of "baker's maps" which act in a Euclidean space of arbitrarily high dimension and have the property that $D_{0}=D_{1}=D_{2}$. An important point is that we allow different branches of the map to overlap in a way which can cause the dimension of the attractor to be less than the conjectured value in special cases; we prove that these exceptional cases have Lebesgue measure zero in the parameter space of the maps we study.

We are also able to determine $D_{2}$ for almost every attractor of a more general class of attractors of baker's maps for which $D_{0}>D_{1}>D_{2}$. Our results are related to those of Falconer [F1], who determines $D_{0}$ (and the Hausdorff dimension) for almost every attractor in an even more general class. See also [F3] [PW] [S1] [S2] for results on the dimension of attractors of "overlapping" geometric constructions.

We consider a class of "skinny baker's maps", described below, with one expanding direction and $N \geq 1$ contracting directions. Our analysis is primarily in the

Received by the editors June 30, 1995.

1991 Mathematics Subject Classification. Primary 28D20, 28D05; Secondary 60G18.

(c)1997 American Mathematical Society 
space of contracting directions, in which we think of the maps as "iterated function systems". Generally speaking, an iterated function system (IFS) is a set of $k$ contracting maps $T_{i}$ (where $i=1, \ldots, k$ ) with associated probabilities $p_{i}$. An iteration of the IFS consists of randomly choosing one of the $T_{i}$ 's and applying it. The IFS studied in this paper will always be applied to a unit cube in $\mathbf{R}^{N}$, and the $T_{i}$ 's will be affine maps and will always have equal probability, i.e., $p_{i}=1 / k$ for all $i=1, \ldots, k$. Further, we assume that each $T_{i}$ consists only of contractions in the directions of the coordinate axes and a translation, and that all contraction rates are bounded above by $1 / 2$. The corresponding skinny baker's map transforms the unit cube in $\mathbf{R}^{N+1}$ into itself: The cube is divided into $k$ equal pieces along the $(N+1)$ th direction, so that each piece has height $1 / k$. For $i=1, \ldots, k$, the $i$ th piece is stretched by a factor of $k$ along the $(N+1)$ th direction to fill the cube, and shrunk in the other directions according to $T_{i}$. Details of the skinny baker's map and its correspondence to an IFS will be given in $\S 2.2$. Note that repeated iterations of an IFS (or a skinny baker's map) not only produces a self-affine fractal, but also gives rise to an invariant probability measure supported on this fractal set.

We provide some background material and introduce the notation we use in $\S 2$ of this paper. We show in $\S 3$ that for the type of IFS we study with a given set of contraction rates, the correlation dimension of the attractor almost always assumes the same value, which is determined solely by the contraction rates. Moreover, we show that if the IFS has uniform contraction rates in each direction, our results imply the Kaplan-Yorke conjecture for the corresponding baker's maps. A conclusion is presented in $\S 4$.

\section{BACKGROUND AND NOTATION}

In this section we provide some background on the dimensions and the baker's map. We also introduce the notation used in this paper.

2.1. Dimensions of attractors. Consider an attractor $\Lambda$ of a smooth map $f$ : $\mathbf{R}^{N} \rightarrow \mathbf{R}^{N}$. By this we mean that $\Lambda$ is the limit set of the trajectory $\left\{f^{i}(x)\right\}_{i \geq 0}$, where $f^{i+1}(x)=f\left(f^{i}(x)\right)$, for a positive Lebesgue measure set $B$ of initial points $x \in \mathbf{R}^{N}$. For a set $S \subset \mathbf{R}^{N}$ and initial point $x \in B$, define $\mu(x, S)$ to be

$$
\mu(x, S)=\lim _{M \rightarrow \infty} \frac{\sum_{i=1}^{M} \chi\left(f^{i}(x) \mid S\right)}{M},
$$

where

$$
\chi\left(f^{i}(x) \mid S\right)= \begin{cases}1 & \text { if } f^{i}(x) \in S, \\ 0 & \text { if } f^{i}(x) \notin S .\end{cases}
$$

The limit defining $\mu(x, S)$ need not exist for all $x \in B$, but it is generally expected (and known for Axiom A diffeomorphisms) that the limit exists and has the same value for almost every $x \in B$ (with respect to Lebesgue measure); if so, we denote this value by $\mu(S)$ and call $\mu$ the natural measure of the attractor [BR] [Ru] [FOY]. The dimension spectrum $D_{q}$ of the attractor $\Lambda$ is defined in [R] [G] [HP] as follows: Cover $\Lambda$ with a uniform grid of mesh size $\epsilon$. Denoting the natural measure in the $j$-th box of the grid by $\mu_{j}$, the dimension spectrum is given by

$$
D_{q}(\Lambda)=\frac{1}{q-1} \lim _{\epsilon \rightarrow 0} \frac{\log \sum_{j=1}^{K} \mu_{j}^{q}}{\log \epsilon},
$$


provided the limit exists. Here $K$ is the number of boxes of size $\epsilon$ needed to cover the set $\Lambda$. The values of $D_{0}, D_{1}$ and $D_{2}$ are called the box-counting (or capacity) dimension, the information dimension, and the correlation dimension respectively ${ }^{1}$.

The Kaplan-Yorke conjecture states that for an attractor of a dynamical system with natural measure $\mu$ and Lyapunov numbers $\lambda_{1} \geq \lambda_{2} \geq \cdots \geq \lambda_{N}$, the information dimension $D_{1}$ of $\mu$ is typically equal to the Lyapunov dimension $D_{L}$, which is given by

$$
D_{L}=m+\frac{\log \left(\lambda_{1} \lambda_{2} \ldots \lambda_{m}\right)}{\left|\log \lambda_{m+1}\right|}
$$

where $m$ is the largest integer such that $\lambda_{1} \lambda_{2} \ldots \lambda_{m} \geq 1$ [KY] [FOY]. This conjecture was proved by Young $[\mathrm{Y}]$ for Axiom A diffeomorphisms in dimension two. However there has been no proof for the conjecture in higher dimensions.

Our main theorem, proved in $\S 3$, similarly gives a dynamical characterization of the correlation dimension $D_{2}$ of the natural measure which holds for "almost every" skinny baker's map. However, our result indicates that the correlation dimension is determined by the Lyapunov numbers of all trajectories, instead of by the Lyapunov numbers of a typical trajectory. In particular, two different sets of contraction rates may yield the same set of Lyapunov numbers for a typical trajectory, but they result in different values of the correlation dimension for the attractor.

Define the correlation integral $C(\delta)$ to be the probability that a pair of points chosen randomly (with respect to the natural measure) are separated by a distance less than $\delta$ on the attractor. Then for a typical trajectory $\left\{f^{i}(x)\right\}_{i \geq 0}$, one expects that

$$
C(\delta)=\lim _{M \rightarrow \infty} \frac{1}{M^{2}} \sum_{i=1}^{M} \sum_{j=1}^{M} \theta\left(\delta-\left|f^{i}(x)-f^{j}(x)\right|\right)_{i \neq j},
$$

where $\theta(\cdot)$ is the Heaviside function, i.e., $\theta(z)=1$ if $z \geq 0$ and $\theta(z)=0$ if $z<0$. The correlation integral $C(\delta)$ often behaves as a power of $\delta$ for small $\delta[\mathrm{GP}][\mathrm{P}]$ :

$$
C(\delta) \sim \delta^{\nu} .
$$

In this case we can write

$$
D_{2}=\nu=\lim _{\delta \rightarrow 0} \frac{\log C(\delta)}{\log \delta} .
$$

This formulation is commonly used to estimate $D_{2}$ from a time series.

The relation between $D_{2}$ and $C(\delta)$ also leads to an alternative definition of the correlation dimension [DGOSY] [SY]

$$
D_{2}(\Lambda)=\sup \left\{s: \int_{\Lambda} \int_{\Lambda} \frac{d \mu(x) d \mu(y)}{|x-y|^{s}}<\infty\right\}=\inf \left\{s: \int_{\Lambda} \int_{\Lambda} \frac{d \mu(x) d \mu(y)}{|x-y|^{s}}=\infty\right\}
$$

where $\mu$ is the natural measure supported on $\Lambda$, and $|x-y|$ is the distance between two points $x$ and $y$ in $\Lambda^{2}$. This is the definition of $D_{2}$ which we use in this paper;

\footnotetext{
${ }^{1} D_{1}$ can be defined as the limit of $D_{q}$ as $q \rightarrow 1$, again provided this limit exists.

${ }^{2}$ Of course, this definition and the previous ones can be made for arbitrary probability measures $\mu$.
} 


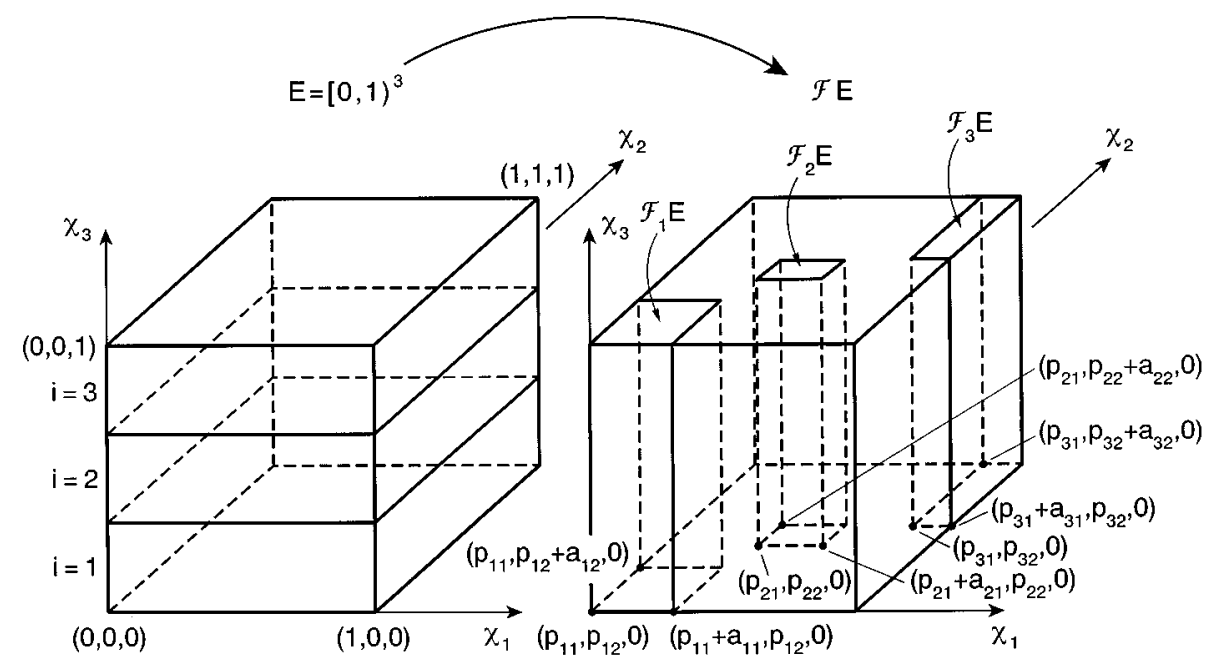

Figure 1. The action of one iteration of the baker's map in $\mathbf{R}^{3}$ for $k=3$.

it is equivalent to (1) and (5) in the case when the limits exist, and more generally corresponds to using a "liminf" in each of the previous definitions. The integral

$$
I_{s}(\Lambda)=\int_{\Lambda} \int_{\Lambda} \frac{d \mu(x) d \mu(y)}{|x-y|^{s}}
$$

is called the $s$-energy of the set $\Lambda$ with respect to the measure $\mu$ [F2]. Since the integrand in $I_{s}(\Lambda)$ is an increasing function of $s$ when $|x-y|<1$, the supremum and the infimum in (6) are equal.

To define the Hausdorff dimension, we cover the set $\Lambda$ by a collection of open sets $\left\{U_{j}: U_{j} \subset \mathbf{R}^{N}\right.$ and $\left.0<\left|U_{j}\right| \leq \delta\right\}$, where $\left|U_{j}\right|=\max \left\{\left|u_{1}-u_{2}\right|: u_{1}, u_{2} \in U_{j}\right\}$ is the diameter of the set. For $0 \leq s \leq n$, write

$$
\mathcal{H}_{\delta}^{s}(\Lambda)=\inf \left\{\sum_{j=1}^{\infty}\left|U_{j}\right|^{s}: \Lambda \subset \bigcup_{j=1}^{\infty} U_{j}, 0<\left|U_{j}\right| \leq \delta\right\},
$$

then $\mathcal{H}^{s}(\Lambda)=\lim _{\delta \rightarrow 0} \mathcal{H}_{\delta}^{s}(\Lambda)$ is called the $s$-dimensional Hausdorff measure of the set $\Lambda$. The Hausdorff dimension is defined as [F2]

$$
H D(\Lambda)=\inf \left\{s: \mathcal{H}^{s}(\Lambda)=0\right\}=\sup \left\{s: \mathcal{H}^{s}(\Lambda)=\infty\right\} .
$$

Notice that unlike the correlation dimension of an attractor, the Hausdorff dimension depends only on the set $\Lambda$ and not on the natural measure induced by the dynamics.

2.2. The baker's map and its correspondence to an IFS. Let $\mathbf{x}=\left(x_{1}, \ldots\right.$, $x_{N+1}$ ) represent a point in $\mathbf{R}^{N+1}$ and $\hat{\mathbf{x}}_{j}$ (where $j=1, \ldots, N+1$ ) be a unit vector in the direction of the $j$-th coordinate. The baker's map $\mathcal{F}$ on the unit cube 
$E=[0,1)^{N+1}$ in $\mathbf{R}^{N+1}$ is defined as follows:

$$
\left\{\begin{array}{cl}
x_{j} & \rightarrow a_{i j} x_{j}+p_{i j} \quad \text { where } i=\left[k x_{N+1}\right]+1 \text { and } j=1, \ldots, N \\
x_{N+1} & \rightarrow k x_{N+1}(\bmod 1) .
\end{array}\right.
$$

Here [ $\cdot]$ is the greatest integer function. The set of values of $k,\left\{a_{i j}\right\}$, and $\left\{p_{i j}\right\}$ are the parameter values, with $0 \leq p_{i j} \leq 1-a_{i j}$ for all $i, j$. Under one iteration of the baker's map, the unit cube $E$ is divided into $k$ equal pieces (each of width $1 / k$ ) along the $\hat{\mathbf{x}}_{N+1}$ direction, each piece carrying an equal weight of $1 / k$. A piece is labeled with an integer " $i$ " if every point it contains has its $x_{N+1}$ coordinate satisfying $i=\left[k x_{N+1}\right]+1$. Thus $i=1, \ldots, k$. Let $\mathcal{F}_{i}$ denote the transformation defined by (10) for fixed $i$, then the image of the $i$-th piece under (10) is given by $\mathcal{F}_{i} E$. Note that $\mathcal{F}_{i} E$ is a column of unit length (in the direction of $\hat{\mathbf{x}}_{N+1}$ ) which occupies the interval $\left[p_{i j}, p_{i j}+a_{i j}\right)$ in the direction of $\hat{\mathbf{x}}_{j}$ for $j=1, \ldots, N$. Moreover, $\mathcal{F} E=\bigcup_{i=1}^{k} \mathcal{F}_{i} E \subset E$. Figure 1 demonstrates the action of one iteration of the baker's map in $\mathbf{R}^{3}$ for $k=3$. For the baker's map, the Lyapunov number in the direction of $\hat{\mathbf{x}}_{j}$ for $j=1, \ldots, N$ is $\lambda_{j}=\left(a_{1 j} a_{2 j} \ldots a_{k j}\right)^{1 / k}$, and the Lyapunov number in the direction of $\hat{\mathbf{x}}_{N+1}$ is $\lambda_{N+1}=k$.

The mapping defined in (10) is called the skinny baker's map if $\max \left\{a_{i j}\right\}<$ $1 / 2$; otherwise it is called the fat baker's map. Complications rooted in number theoretical difficulties arise in computations of the dimensions for the fat baker's map $[\mathrm{AY}][\mathrm{PoW}]$. In this work, we only consider the skinny baker's map, i.e., we assume $\max \left\{a_{i j}\right\}<1 / 2$ in (10).

Denote $x^{(N+1)}=\left(x_{1}, \ldots, x_{N}, x_{N+1}\right) \in \mathbf{R}^{N+1}$ and $y^{(N)}=\left(y_{1}, \ldots, y_{N}\right) \in \mathbf{R}^{N}$. (In places where no confusion is caused, we omit the superscripts $(N+1)$ and $(N)$ in $x^{(N+1)}$ and $y^{(N)}$.) We define a perpendicular projection $\Pi_{N}^{N+1}: \mathbf{R}^{N+1} \rightarrow \mathbf{R}^{N}$ to be $\Pi_{N}^{N+1}\left(x^{(N+1)}\right)=y^{(N)}$, such that $x_{i}=y_{i}$ for $i=1, \ldots, N$. For a set $S \subset \mathbf{R}^{N+1}$, $\Pi_{N}^{N+1}(S)=\left\{\Pi_{N}^{N+1}\left(x^{(N+1)}\right): x^{(N+1)} \in S\right\} \subset \mathbf{R}^{N}$. Hence for the unit cube $E=$ $[0,1)^{N+1}$ in $\mathbf{R}^{N+1}, Q=\Pi_{N}^{N+1}(E)$ is the unit cube $[0,1)^{N}$ in $\mathbf{R}^{N}$.

We denote the map in $\mathbf{R}^{N}$

$$
x_{j} \rightarrow a_{i j} x_{j}+p_{i j}
$$

in (10) for fixed $i$ by $T_{i}$, where $j=1, \ldots, N$. Let $T$ denote the set function $\bigcup_{i=1}^{k} T_{i}$; that is, $T(S)=\bigcup_{i=1}^{k} T_{i}(S)$ for all sets $S \subset Q$. Then $\Pi_{N}^{N+1}\left(\mathcal{F}^{n} E\right)=T^{n} Q$ for all $n$. Here $\mathcal{F}^{n+1} E=\mathcal{F}\left(\mathcal{F}^{n} E\right)$ and $T^{n+1} Q=T\left(T^{n} Q\right)$. The box given by $T_{i} Q=$ $\Pi_{N}^{N+1}\left(\mathcal{F}_{i} E\right)$ in $\mathbf{R}^{N}$ is labeled with " $i$ ", since the column $\mathcal{F}_{i} E$ in $\mathbf{R}^{N+1}$ is labeled with " $i$ ". Figure 2 demonstrates the action of one iteration of the map $T$ in $\mathbf{R}^{2}$ with $k=3$. If we let the map $T$ in Figure 2 have the same set of values of $\left\{a_{i j}\right\}$ and $\left\{p_{i j}\right\}$ as the map $\mathcal{F}$ in Figure 1, where $i=1,2,3$ and $j=1,2$, then the set $T Q$ in Figure 2 is the vertical projection of the set $\mathcal{F} E$ in Figure 1 onto the $x_{1} x_{2}$ plane.

Under the baker's transformation defined in (10), an invariant set $\Lambda$ exists within the unit cube $E=[0,1)^{N+1}$, which is given by

$$
\Lambda=\bigcap_{n=1}^{\infty} \mathcal{F}^{n} E .
$$




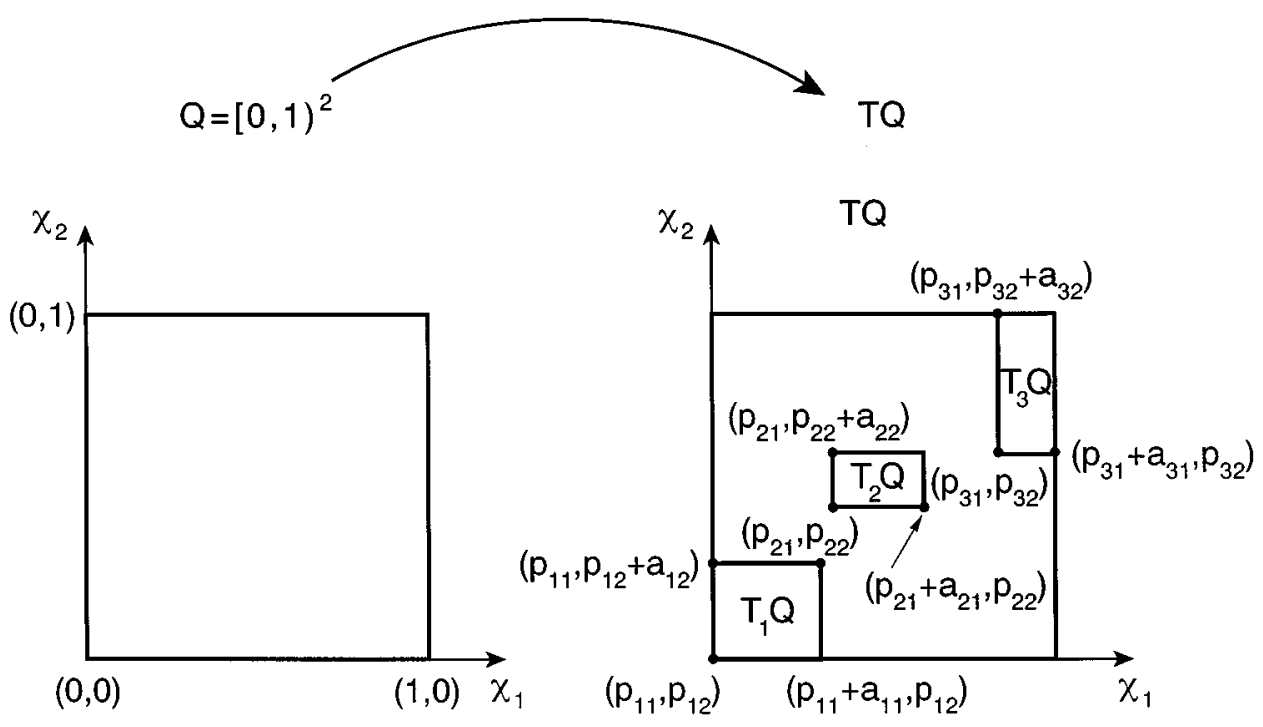

Figure 2. The action of one iteration of the map $T$ in $\mathbf{R}^{2}$ with $k=3$.

Similarly, under the map $T$, an invariant set $A$ exists in the unit cube $Q=[0,1)^{N}$, and is given by

$$
A=\bigcap_{n=1}^{\infty} T^{n} Q .
$$

The sets $\mathcal{F}^{n} E$ and $T^{n} Q$ are also denoted by $\Lambda_{n}$ and $A_{n}$ respectively. Notice that, $A=\Pi_{N}^{N+1} \Lambda$ and $A_{n}=\Pi_{N}^{N+1} \Lambda_{n}$ for all $n$. To each $A_{n}$ we assign a probability measure $\mu_{n}$ consisting of the average of the uniform probability measures on the $k^{n}$ boxes $T_{i_{1}} T_{i_{2}} \cdots T_{i_{n}} Q$ which make up $A_{n}$. We say that the natural measure $\mu$ of $A$ is the limit of the measures $\mu_{n}$. Then the natural measure of the attractor $\Lambda$ of $\mathcal{F}$ is the product of $\mu$ with a uniform measure in the direction of $\hat{\mathbf{x}}_{N+1}$. In particular, the dimension of $A$ is one less than that of $\Lambda$.

From the discussions of the iterated function systems in $\S 1$, we have that the IFS corresponding to the skinny baker's map above is to apply each $T_{i}$ with probability $1 / k$, and that $T$ represents the union of all possible outcomes of one iterate of the IFS. Then the projection of the dynamics of the skinny baker's map onto $\mathbf{R}^{N}$ corresponds to the IFS determined by $T$. In particular, the attractor $A$ given in (13) is also the attractor for the corresponding IFS, and they have the same natural invariant measure $\mu$.

2.3. Notation. We adopt the notation used in [F1].

Recall that in the $x_{1} \ldots x_{N}$ hyperplane, $T Q=\bigcup_{i=1}^{k} T_{i} Q$ where $Q=[0,1)^{N}$. We label the box $T_{i} Q$ by " $i$ ". At the next level, $T^{2} Q=\bigcup_{i, j=1}^{k} T_{j} T_{i} Q$. Thus we label the box $T_{j} T_{i} Q$ by " $j i$ ", where $i, j=1, \ldots, k$. Continuing in this fashion, we label a box in $T^{n} Q$ with a symbol sequence of length $n$ consisting of the symbols "1", ..., " $k$ ". Let us denote the set of symbol sequences of length $n$ consisting of the symbols " $1 ", \ldots$, " $k$ " by $S_{k}^{n}$, and the set of infinite symbol sequences consisting of the symbols " 1 ", ..., " $k$ " by $S_{k}^{\infty}$. For $\mathbf{c} \in S_{k}^{n}$ where $n>1, c_{i}$ is the $i$-th symbol 
of the sequence $\mathbf{c}$, where $i \leq n$. Note that each box in $T^{n} Q$ corresponds to a distinct element in $S_{k}^{n}$ and vice versa. Now we assign to each distinct infinite symbol sequence in $S_{k}^{\infty}$ a point $x \in A$ (where $A$ is the invariant set defined in (13)) in the following way. Given $\mathbf{c}$ in $S_{k}^{\infty}$, the boxes corresponding to finite truncations of $\mathbf{c}$ form a nested sequence of sets whose intersection is a single point which we denote $x_{\mathbf{c}}(\mathbf{p})$. Here the vector $\mathbf{p} \in \mathbf{R}^{N k}$ is given by

$$
\mathbf{p}=\left(p_{11}, \ldots, p_{1 N}, p_{21}, \ldots, p_{2 N}, \ldots, p_{k 1}, \ldots, p_{k N}\right) \in B,
$$

where the components $\left\{p_{i j}\right\}$ with $i=1, \ldots, k$ and $j=1, \ldots, N$ are given in (10), and $B$ is the box $\left\{0 \leq p_{i j} \leq 1-a_{i j}\right\}$ in $\mathbf{R}^{N k}$. Note that depending on the values of $\left\{p_{i j}\right\}$, there may be more than one infinite sequence in $S_{k}^{\infty}$ that correspond to the same point in $A$.

Let $\mathbf{b}, \mathbf{b}^{\prime}$ be the sequence obtained by juxtaposition of the terms of $\mathbf{b}$ and $\mathbf{b}^{\prime}$, where $\mathbf{b} \in S_{k}^{n}$ for some $n<\infty$. If $\mathbf{b}$ is a curtailment of $\mathbf{c}$, that is if $\mathbf{c}=\mathbf{b}, \mathbf{b}^{\prime}$ for some $\mathbf{b}^{\prime}$, we write $\mathbf{b}<\mathbf{c}$. If $\mathbf{c}$ and $\mathbf{e}$ are elements of $S_{k}^{\infty}$ then $\mathbf{c} \wedge \mathbf{e}$ is the maximal sequence such that $\mathbf{c} \wedge \mathbf{e}<\mathbf{c}$ and $\mathbf{c} \wedge \mathbf{e}<\mathbf{e}$. Consider the set $N_{\mathbf{b}}=\left\{\mathbf{c} \in S_{k}^{\infty}: \mathbf{c}>\mathbf{b}\right\} \subset S_{k}^{\infty}$. Since all sequences of a given length are equally likely, we define the "uniform" measure $\nu$ on $S_{k}^{\infty}$ such that $\nu\left(N_{\mathbf{b}}\right)=k^{-|\mathbf{b}|}$, where $|\mathbf{b}|$ is the length of $\mathbf{b}$. Then the natural measure $\mu$ on $A$ is the image of $\nu$ under the correspondence $\mathbf{c} \rightarrow x_{\mathbf{c}}(\mathbf{p})$. In this notation, the $s$-energy defined in Eq. (7) takes the form

$$
\begin{aligned}
I_{s}(A) & =\int_{S_{k}^{\infty}} \int_{S_{k}^{\infty}} \frac{d \nu(\mathbf{c}) d \nu(\mathbf{e})}{\left|x_{\mathbf{c}}(\mathbf{p})-x_{\mathbf{e}}(\mathbf{p})\right|^{s}} \\
& =\sum_{r=1}^{\infty} \sum_{\mathbf{q} \in S_{k}^{r}} \int_{\mathbf{c} \wedge \mathbf{e}=\mathbf{q}} \frac{d \nu(\mathbf{c}) d \nu(\mathbf{e})}{\left|x_{\mathbf{c}}(\mathbf{p})-x_{\mathbf{e}}(\mathbf{p})\right|^{s}} .
\end{aligned}
$$

Now consider the box in $Q$ which is given by $T_{b_{1}} \ldots T_{b_{n}} Q$ (and thus has symbol sequence $\left.\mathbf{b}=b_{1} \ldots b_{n}\right)$, where $T_{i}$ is defined in (11) for $i=1, \ldots, k$. We denote the composite mapping $T_{b_{1}} \ldots T_{b_{n}}$ by $T_{\mathbf{b}}$. We define the singular values $\alpha_{1}^{(\mathbf{b})}, \ldots, \alpha_{N}^{(\mathbf{b})}$ to be the resultant contractions of $T_{\mathbf{b}}$ in the directions of $\hat{\mathbf{x}}_{1}, \ldots, \hat{\mathbf{x}}_{N}$ respectively, that is, $\alpha_{j}^{(\mathbf{b})}=a_{b_{1} j} \ldots a_{b_{n} j}$ for $j=1, \ldots, N$. We re-label the values $\alpha_{1}^{(\mathbf{b})}, \ldots, \alpha_{N}^{(\mathbf{b})}$ by $\alpha_{[1]}^{(\mathbf{b})}, \alpha_{[2]}^{(\mathbf{b})}, \ldots, \alpha_{[N]}^{(\mathbf{b})}$ so that $\alpha_{[1]}^{(\mathbf{b})} \geq \alpha_{[2]}^{(\mathbf{b})} \geq \cdots \geq \alpha_{[N]}^{(\mathbf{b})}$. Then corresponding to a composite mapping $T_{\mathbf{b}}$, the singular value function $\phi^{s}\left(T_{\mathbf{b}}\right)$ is defined as

$$
\phi^{s}\left(T_{\mathbf{b}}\right)=\alpha_{[1]}^{(\mathbf{b})} \alpha_{[2]}^{(\mathbf{b})} \cdots \alpha_{[m-1]}^{(\mathbf{b})}\left(\alpha_{[m]}^{(\mathbf{b})}\right)^{s-m+1},
$$

where $m$ is the integer satisfying $m-1<s \leq m$. We write

$$
\phi^{s}\left(T_{\mathbf{b}}\right)=\left(\alpha_{[1]}^{(\mathbf{b})} \cdots \alpha_{[N]}^{(\mathbf{b})}\right)^{s / N}
$$

if $s>N$. Notice that $\phi^{s}\left(T_{\mathbf{b}}\right)$ is a strictly decreasing function of $s$.

\section{LOWER AND UPPER BOUNDS OF CORRELATION DIMENSION}

Our main theorem is as follows:

Main theorem. For the skinny baker's map defined in (10), let the values of $k$ and $\left\{a_{i j}\right\}$ be given. The correlation dimension of the invariant set $A$ (as defined in (13)) has the same value, determined solely by the values of $k$ and $\left\{a_{i j}\right\}$, for almost all sets of $\left\{p_{i j}\right\}$. 
We show this by assessing the lower and upper bounds of the correlation dimension $D_{2}(A)$. From the definition of the correlation dimension in Eq. (6), we observe:

(i) Lower bound condition: If $I_{s}(A)<\infty$, then $D_{2}(A) \geq s$.

(ii) Upper bound condition: If $I_{s}(A)=\infty$, then $D_{2}(A) \leq s$.

3.1. Lower bound. Let $B$ be the box $\left\{0 \leq p_{i j} \leq 1-a_{i j}\right\}$ in $\mathbf{R}^{N k}$, and let $\mathbf{p} \in \mathbf{R}^{N k}$ be given by (14). The lower bound condition (i) indicates that $s$ is a lower bound of $D_{2}(A)$ for almost all sets of $\left\{p_{i j}\right\}$ (where $\left.i=1, \ldots, k, j=1, \ldots, N\right)$ if

$$
\int_{\mathbf{p} \in B} I_{s}(A) d \mathbf{p}<\infty
$$

because this inequality implies that the correlation integral $I_{s}(A)$ is finite for almost all $\mathbf{p} \in B$.

Using the result stated in Lemma 3.1 of [F1], we obtain a series that bounds the integral in (17) from above, and which depends only on the values of $k$ and $\left\{a_{i j}\right\}$ in (10). Letting $\mathbf{c} \in S_{k}^{\infty}, \mathbf{e} \in S_{k}^{\infty}$, and $\mathbf{q}=\mathbf{c} \wedge \mathbf{e}$, it follows from [F1] that

$$
\begin{aligned}
\int_{\mathbf{p} \in B} I_{s}(A) d \mathbf{p} & =\int_{\mathbf{p} \in B} \int_{S_{k}^{\infty}} \int_{S_{k}^{\infty}} \frac{d \nu(\mathbf{c}) d \nu(\mathbf{e}) d \mathbf{p}}{\left|x_{\mathbf{c}}(\mathbf{p})-x_{\mathbf{e}}(\mathbf{p})\right|^{s}} \\
& =\int_{S_{k}^{\infty}} \int_{S_{k}^{\infty}} \int_{\mathbf{p} \in B} \frac{d \mathbf{p} d \nu(\mathbf{c}) d \nu(\mathbf{e})}{\left|x_{\mathbf{c}}(\mathbf{p})-x_{\mathbf{e}}(\mathbf{p})\right|^{s}} \\
& \leq \kappa \sum_{r=1}^{\infty} \sum_{\mathbf{q} \in S_{k}^{r}} \phi^{s}\left(T_{\mathbf{q}}\right)^{-1} \nu\left(N_{\mathbf{q}}\right)^{2},
\end{aligned}
$$

where $\kappa$ is a finite constant, and $\phi^{s}\left(T_{\mathbf{q}}\right)$ is the singular value function of the composite mapping $T_{\mathbf{q}}$. Therefore any value of $s$ satisfying

$$
\sum_{r=1}^{\infty} \sum_{\mathbf{q} \in S_{k}^{r}} \phi^{s}\left(T_{\mathbf{q}}\right)^{-1} k^{-2 r}<\infty
$$

is a lower bound of $D_{2}(A)$ for almost all sets of $\left\{p_{i j}\right\}$ (with a given set of $\left\{a_{i j}\right\}$ and $k$ ). Thus

$$
D_{2}(A) \geq \sup \left\{s: \sum_{r=1}^{\infty} \sum_{\mathbf{q} \in S_{k}^{r}} \phi^{s}\left(T_{\mathbf{q}}\right)^{-1} k^{-2 r}<\infty\right\}
$$

for almost all values of $\left\{p_{i j}\right\}$.

3.2. Upper bound. We first prove some results that are needed in finding an upper bound on $D_{2}(A)$.

Proposition 1. Let $s, t \in \mathbf{R}$. If the series

$$
\tilde{I}_{s}(A)=\sum_{r=1}^{\infty} \sum_{\mathbf{q} \in S_{k}^{r}} \int_{\mathbf{c} \wedge \mathbf{e} \geq \mathbf{q}} \frac{d \mu\left(x_{\mathbf{c}}(\boldsymbol{p})\right) d \mu\left(x_{\mathbf{e}}(\boldsymbol{p})\right)}{\left|x_{\mathbf{c}}(\boldsymbol{p})-x_{\mathbf{e}}(\boldsymbol{p})\right|^{s}}
$$

diverges for $s=t$, then the series

$$
I_{s}(A)=\sum_{r=1}^{\infty} \sum_{\mathbf{q} \in S_{k}^{r}} \int_{\mathbf{c} \wedge \mathbf{e}=\mathbf{q}} \frac{d \mu\left(x_{\mathbf{c}}(\boldsymbol{p})\right) d \mu\left(x_{\mathbf{e}}(\boldsymbol{p})\right)}{\left|x_{\mathbf{c}}(\boldsymbol{p})-x_{\mathbf{e}}(\boldsymbol{p})\right|^{s}}
$$

diverges for all $s>t$. 
Proof. Notice that if $\mathbf{c} \wedge \mathbf{e}=\mathbf{q} \in S_{k}^{r}$, then the points $x_{\mathbf{c}}(\mathbf{p})$ and $x_{\mathbf{e}}(\mathbf{p})$ both belong to the box $T_{\mathbf{q}} Q$ at the $r$-th level, but they are in different boxes at the $(r+1)$-th level. That is, $c_{n}=e_{n}$ for $n=1, \ldots, r$, and $c_{r+1} \neq e_{r+1}$. On the other hand, $\mathbf{c} \wedge \mathbf{e} \geq \mathbf{q}$ means that $c_{n}$ may or may not be the same as $e_{n}$ for $n>r$. In this case $x_{\mathbf{c}}(\mathbf{p})$ and $x_{\mathbf{e}}(\mathbf{p})$ are points that both belong to the box $T_{\mathbf{q}} Q$ at the $r$-th level, and they may or may not be in different boxes at higher levels. Thus if $\mathbf{c} \wedge \mathbf{e}=\mathbf{q} \in S_{k}^{r_{0}}$, then the quantity $1 /\left|x_{\mathbf{c}}(\mathbf{p})-x_{\mathbf{e}}(\mathbf{p})\right|^{s}$ is present in the summation in Eq. (21) for $r=1, \ldots, r_{0}$. Therefore,

$$
\sum_{r=1}^{\infty} \sum_{\mathbf{q} \in S_{k}^{r}} \int_{\mathbf{c} \wedge \mathbf{e} \geq \mathbf{q}} \frac{d \mu\left(x_{\mathbf{c}}(\mathbf{p})\right) d \mu\left(x_{\mathbf{e}}(\mathbf{p})\right)}{\left|x_{\mathbf{c}}(\mathbf{p})-x_{\mathbf{e}}(\mathbf{p})\right|^{s}}=\sum_{r=1}^{\infty} r \sum_{\mathbf{q} \in S_{k}^{r}} \int_{\mathbf{c} \wedge \mathbf{e}=\mathbf{q}} \frac{d \mu\left(x_{\mathbf{c}}(\mathbf{p})\right) d \mu\left(x_{\mathbf{e}}(\mathbf{p})\right)}{\left|x_{\mathbf{c}}(\mathbf{p})-x_{\mathbf{e}}(\mathbf{p})\right|^{s}} .
$$

Now suppose that the sum in Eq. (23) diverges for $s=t$. We examine the convergence (divergence) of the sum in Eq. (22) for $s=t+\epsilon$ with $\epsilon>0$ :

$$
\begin{aligned}
& \sum_{r=1}^{\infty} \sum_{\mathbf{q} \in S_{k}^{r}} \int_{\mathbf{c} \wedge \mathbf{e}=\mathbf{q}} \frac{d \mu\left(x_{\mathbf{c}}(\mathbf{p})\right) d \mu\left(x_{\mathbf{e}}(\mathbf{p})\right)}{\left|x_{\mathbf{c}}(\mathbf{p})-x_{\mathbf{e}}(\mathbf{p})\right|^{t+\epsilon}} \\
& \quad=\sum_{r=1}^{\infty} \sum_{\mathbf{q} \in S_{k}^{r}} \int_{\mathbf{c} \wedge \mathbf{e}=\mathbf{q}}\left[\frac{d \mu\left(x_{\mathbf{c}}(\mathbf{p})\right) d \mu\left(x_{\mathbf{e}}(\mathbf{p})\right)}{\left|x_{\mathbf{c}}(\mathbf{p})-x_{\mathbf{e}}(\mathbf{p})\right|^{t}} \cdot \frac{1}{\left|x_{\mathbf{c}}(\mathbf{p})-x_{\mathbf{e}}(\mathbf{p})\right|^{\epsilon}}\right] \\
& \quad \geq \sum_{r=1}^{\infty} 2^{r \epsilon} \sum_{\mathbf{q} \in S_{k}^{r}} \int_{\mathbf{c} \wedge \mathbf{e}=\mathbf{q}} \frac{d \mu\left(x_{\mathbf{c}}(\mathbf{p})\right) d \mu\left(x_{\mathbf{e}}(\mathbf{p})\right)}{\left|x_{\mathbf{c}}(\mathbf{p})-x_{\mathbf{e}}(\mathbf{p})\right|^{t}} \quad \text { since }\left|x_{\mathbf{c}}(\mathbf{p})-x_{\mathbf{e}}(\mathbf{p})\right| \leq 2^{-r} \\
& \quad \geq \sum_{r=1}^{\infty} \beta r \sum_{\mathbf{q} \in S_{k}^{r}} \int_{\mathbf{c} \wedge \mathbf{e}=\mathbf{q}} \frac{d \mu\left(x_{\mathbf{c}}(\mathbf{p})\right) d \mu\left(x_{\mathbf{e}}(\mathbf{p})\right)}{\left|x_{\mathbf{c}}(\mathbf{p})-x_{\mathbf{e}}(\mathbf{p})\right|^{t}} \quad \text { for some } \beta>0 \\
& \quad=\infty . \quad \square
\end{aligned}
$$

Proposition 2. Let $\mu$ be a probability measure supported on the unit cube $E=$ $[0,1)^{N} \subset \mathbf{R}^{N}$. There exists a positive number $\xi$ such that, for any two points $x=\left(x_{1}, \ldots, x_{N}\right) \in E$ and $y=\left(y_{1}, \ldots, y_{N}\right) \in E$, the probability (with respect to the measure $\mu$ ) that $\left|x_{1}-y_{1}\right|<\delta_{1},\left|x_{2}-y_{2}\right|<\delta_{2}, \ldots$, and $\left|x_{N}-y_{N}\right|<\delta_{N}$ is at least $\xi \delta_{1} \delta_{2} \cdots \delta_{N}$.

Proof. Suppose $1 / m_{j} \leq \delta_{j} \leq 1 /\left(m_{j}-1\right)$, where $j=1, \ldots, N$ and $\left\{m_{j}\right\}$ are integers. We divide the unit cube $E=[0,1)^{N} \subset \mathbf{R}^{N}$ into a mesh, so that there are $m_{j}$ divisions in the direction of $\hat{\mathbf{x}}_{j}$, each of length $1 / m_{j}$. Then the total number of boxes in the unit cube is $m_{1} m_{2} \cdots m_{N}$. Let $\mu_{i}$ be the measure contained in the $i$-th mesh, then

$$
\sum_{i=1}^{m_{1} \cdots m_{N}} \mu_{i}=1
$$

For two arbitrary points $x, y \in E$, let $P$ be the probability (with respect to the measure $\mu$ ) that $\left|x_{1}-y_{1}\right|<\delta_{1},\left|x_{2}-y_{2}\right|<\delta_{2}, \ldots$, and $\left|x_{N}-y_{N}\right|<\delta_{N}$. Then $P$ is at least the probability (with respect to the measure $\mu$ ) that two points fall in the 
same mesh in the construction above. That is,

$$
\begin{aligned}
P & \geq \sum_{i=1}^{m_{1} \cdots m_{N}} \mu_{i}^{2} \\
& =\left(\sum_{i=1}^{m_{1} \cdots m_{N}} \mu_{i}\right)^{2}-2 \sum_{i, j=1}^{m_{1} \cdots m_{N}} \mu_{i} \mu_{j} \quad \text { where } i>j \\
& \geq 1-\left(m_{1} \cdots m_{N}-1\right) \sum_{i=1}^{m_{1} \cdots m_{N}} \mu_{i}^{2} \\
& \geq 1-\left(m_{1} \cdots m_{N}-1\right) P . \quad \square
\end{aligned}
$$

Thus we have

$$
P \geq \frac{1}{m_{1} \cdots m_{N}} \geq \frac{2^{N}}{\left(m_{1}-1\right) \cdots\left(m_{N}-1\right)} \geq 2^{N} \delta_{1} \cdots \delta_{N} .
$$

We seek an upper bound of the correlation dimension $D_{2}(A)$ by constructing a series (dependent only on $k$ and the contraction rates $\left\{a_{i j}\right\}$ ) that is bounded by $I_{s}(A)$ from above. The values of $s$ that cause the constructed series to diverge are upper bounds of $D_{2}(A)$ for any set of $\left\{p_{i j}\right\}$. We will find an upper bound of $D_{2}(A)$ using this method for the skinny baker's map in $\mathbf{R}^{2+1}$ with $k=3$. The same method applies for the skinny baker's map in $\mathbf{R}^{N+1}$ for arbitrary values of $k$, and the generalization is left to the reader.

Recall from Eqs. (7) and (15) that

$$
\begin{aligned}
I_{s}(A) & =\int_{A} \int_{A} \frac{d \mu(x) d \mu(y)}{|x-y|^{s}} \\
& =\sum_{r=1}^{\infty} \sum_{\mathbf{q} \in S_{k}^{r}} \int_{\mathbf{c} \wedge \mathbf{e}=\mathbf{q}} \frac{d \mu\left(x_{\mathbf{c}}(\mathbf{p})\right) d \mu\left(x_{\mathbf{e}}(\mathbf{p})\right)}{\left|x_{\mathbf{c}}(\mathbf{p})-x_{\mathbf{e}}(\mathbf{p})\right|^{s}} .
\end{aligned}
$$

Notice that for $\mathbf{c} \wedge \mathbf{e}=\mathbf{q} \in S_{k}^{r}$, the points $x_{\mathbf{c}}(\mathbf{p})$ and $x_{\mathbf{e}}(\mathbf{p})$ are both in the box given by $T_{\mathbf{q}} Q$ at the $r$-th level, but are in different boxes at the $(r+1)$-th level. Let $\mathbf{q}=q_{1} q_{2} \ldots q_{r} ;$ then the box $T_{\mathbf{q}} Q$ has sides $\alpha_{1}^{(\mathbf{q})}=a_{q_{1} 1} \ldots a_{q_{r} 1}$ and $\alpha_{2}^{(\mathbf{q})}=a_{q_{1} 2} \ldots a_{q_{r} 2}$. Write $\alpha_{[1]}^{(\mathbf{q})}=\max \left\{\alpha_{1}^{(\mathbf{q})}, \alpha_{2}^{(\mathbf{q})}\right\}$ and $\alpha_{[2]}^{(\mathbf{q})}=\min \left\{\alpha_{1}^{(\mathbf{q})}, \alpha_{2}^{(\mathbf{q})}\right\}$. Then for any $\mathbf{c}$ and $\mathbf{e}$ satisfying $\mathbf{c} \wedge \mathbf{e}=\mathbf{q}$, we have $\left|x_{\mathbf{c}}(\mathbf{p})-x_{\mathbf{e}}(\mathbf{p})\right| \leq \sqrt{2} \alpha_{[1]}^{(\mathbf{q})}$. From this estimation we obtain a lower bound of the correlation integral

$$
I_{s}(A) \geq h \sum_{r=1}^{\infty} \sum_{\mathbf{q} \in S_{k}^{r}} \frac{\mu\left(N_{\mathbf{q}}\right)^{2}}{\left(\alpha_{[1]}^{(\mathbf{q})}\right)^{s}}=h \sum_{r=1}^{\infty} \sum_{\mathbf{q} \in S_{k}^{r}} \frac{1}{k^{2 r}\left(\alpha_{[1]}^{(\mathbf{q})}\right)^{s}},
$$

where $h$ is a positive constant. Any value of $s$ causing the double sum in (27) to diverge is an upper bound of $D_{2}(A)$ for all sets of $\left\{p_{i j}\right\}$.

Now consider the series $\tilde{I}_{s}(A)$ defined in $(21)$. If $\tilde{I}_{s}(A)$ diverges for $s=t$, then by Proposition $1, I_{s}(A)$ diverges for all $s>t$. This makes $t$ an upper bound of $D_{2}(A)$ for all sets of $\left\{p_{i j}\right\}$ (with fixed values of $k$ and $\left\{a_{i j}\right\}$ ). We are able to obtain another estimate of the upper bound of $D_{2}(A)$ via this avenue. In some cases, this new estimation gives a smaller upper bound of $D_{2}(A)$ than the one obtained from (27). 


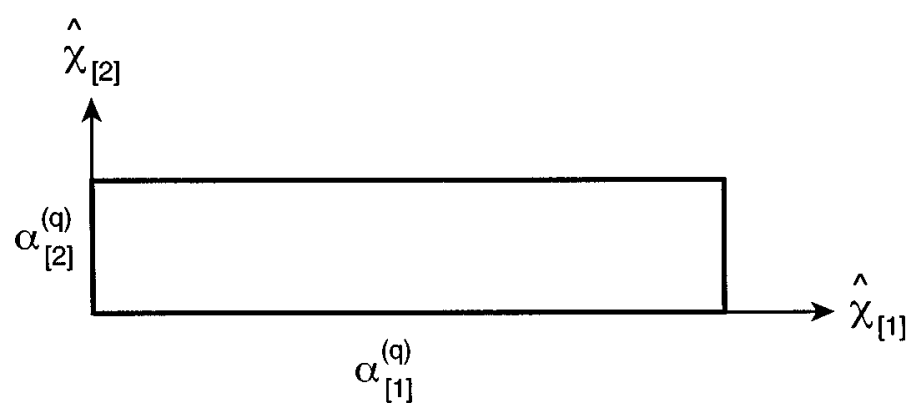

FiguRE 3. Schematic drawing of the box $T_{\mathbf{q}} Q$.

Let $0<f<1$ be the probability that two arbitrary points in $T_{\mathbf{q}} A$ (chosen with respect to $\mu$ ) have distance $\sqrt{2} \alpha_{[2]}^{(\mathbf{q})}$ or less. Then,

$$
\tilde{I}_{s}(A) \geq \sum_{r=1}^{\infty} \sum_{\mathbf{q} \in S_{k}^{r}} \mu\left(N_{\mathbf{q}}\right)^{2}\left(\frac{f}{\left(\sqrt{2} \alpha_{[2]}^{(\mathbf{q})}\right)^{s}}\right)=\sum_{r=1}^{\infty} \sum_{\mathbf{q} \in S_{k}^{r}} k^{-2 r} \frac{f}{\left(\sqrt{2} \alpha_{[2]}^{(\mathbf{q})}\right)^{s}} .
$$

The set $T_{\mathbf{q}} A$ is contained in the box $T_{\mathbf{q}} Q$ which has sides $\alpha_{[1]}^{(\mathbf{q})}$ and $\alpha_{[2]}^{(\mathbf{q})}$. We label the direction the side $\alpha_{[1]}^{(\mathbf{q})}$ is in by a unit vector $\hat{\mathbf{x}}_{[1]}$, and the other direction by a unit vector $\hat{\mathbf{x}}_{[2]}$, as shown in Figure 3 . Two points in the box $T_{\mathbf{q}} Q$ have distance $\sqrt{2} \alpha_{[2]}^{(\mathbf{q})}$ or less if their distance in the $\hat{\mathbf{x}}_{[1]}$ direction is $\alpha_{[2]}^{(\mathbf{q})}$ or less. Notice that stretching the set $T_{\mathbf{q}} A$ by a factor of $1 / \alpha_{[1]}^{(\mathbf{q})}$ in the direction of $\hat{\mathbf{x}}_{[1]}$, and by a factor of $1 / \alpha_{[2]}^{(\mathbf{q})}$ in the direction of $\hat{\mathbf{x}}_{[2]}$ reproduces the invariant set $A$. Hence the value of $f$ in (28) is at least the probability that two points in $A$ have distance less than $\alpha_{[2]}^{(\mathbf{q})} / \alpha_{[1]}^{(\mathbf{q})}$ in the direction of $\hat{\mathbf{x}}_{[1]}$, and distance less than 1 in the direction of $\hat{\mathbf{x}}_{[2]}$. By Proposition 2 there exists a positive number $\xi$ such that $f \geq \xi \alpha_{[2]}^{(\mathbf{q})} / \alpha_{[1]}^{(\mathbf{q})}$. Therefore there is a positive number $h$ so that

$$
\tilde{I}_{s}(A) \geq h \sum_{r=1}^{\infty} \sum_{\mathbf{q} \in S_{k}^{r}} k^{-2 r} \frac{1}{\alpha_{[1]}^{(\mathbf{q})}\left(\alpha_{[2]}^{(\mathbf{q})}\right)^{s-1}} .
$$

A value of $s$ causing the double sum in (29) to diverge is also an upper bound of $D_{2}(A)$.

Recall from (16) that $\phi^{s}\left(T_{\mathbf{q}}\right)=\left(\alpha_{[1]}^{(\mathbf{q})}\right)^{s}$ for $s<1$ and $\phi^{s}\left(T_{\mathbf{q}}\right)=\alpha_{[1]}^{(\mathbf{q})}\left(\alpha_{[2]}^{(\mathbf{q})}\right)^{s-1}$ for $s>1$. Combining (27) and (29), we have

$$
\tilde{I}_{s}(A) \geq h \sum_{r=1}^{\infty} \sum_{\mathbf{q} \in S_{k}^{r}} k^{-2 r} \phi^{s}\left(T_{\mathbf{q}}\right)^{-1}
$$

Thus

$$
D_{2}(A) \leq \inf \left\{s: \sum_{r=1}^{\infty} \sum_{\mathbf{q} \in S_{k}^{r}} k^{-2 r} \phi^{s}\left(T_{\mathbf{q}}\right)^{-1}=\infty\right\}
$$

for all values of $\left\{p_{i j}\right\}$. 
Combining (20) and (31), we conclude that

$$
\begin{aligned}
D_{2}(A) & =\sup \left\{s: \sum_{r=1}^{\infty} \sum_{\mathbf{q} \in S_{k}^{r}} \phi^{s}\left(T_{\mathbf{q}}\right)^{-1} k^{-2 r}<\infty\right\} \\
& =\inf \left\{s: \sum_{r=1}^{\infty} \sum_{\mathbf{q} \in S_{k}^{r}} \phi^{s}\left(T_{\mathbf{q}}\right)^{-1} k^{-2 r}=\infty\right\} .
\end{aligned}
$$

for almost all values of $\left\{p_{i j}\right\}$.

3.3. The case of uniform contraction. In the case where the contraction is uniform in each direction, i.e., $T_{1}, T_{2}, \ldots, T_{N}$ are identical except for translations, we have the following corollary (this corollary is a generalization of Theorem 3 in $[\mathrm{GH}])$ :

Corollary. Assume $a_{i j}=a_{j}$ for each $i, j$ in (10) and (11). Then for almost every set $\left\{p_{i j}\right\}$ in (10) and (11), $D_{0}(A)=D_{1}(A)=D_{2}(A)$ and $D_{1}(\Lambda)=D_{1}(A)+1=$ $D_{L}(\Lambda)$, where $\Lambda$ is given in (12).

Proof. In this case $\phi^{s}\left(T_{\mathbf{q}}\right)$ depends only on $|\mathbf{q}|$, and we can find explicit formulas for $D_{0}(A)$ and $D_{2}(A)$. Since in general $D_{0}(A) \geq D_{1}(A) \geq D_{2}(A)$, once we show that $D_{0}(A)=D_{2}(A)$ then $D_{1}(A)$ must have the same value. For clarity, we again restrict to the case $N=2$, and leave the generalization to the reader.

Suppose $a_{1}>a_{2}$, then we have $\phi^{s}\left(T_{\mathbf{q}}\right)=a_{1}^{|\mathbf{q}| s}$ for $s<1$, and $\phi^{s}\left(T_{\mathbf{q}}\right)=$ $a_{1}^{|\mathbf{q}|} a_{2}^{|\mathbf{q}|(s-1)}$ for $1<s<2$. We write $\psi^{s}\left(a_{1}, a_{2}\right)=a_{1}^{s}$ for $s<1$ and $\psi^{s}\left(a_{1}, a_{2}\right)=$ $a_{1} a_{2}^{s-1}$ for $1<s<2$. Substituting these into the result on box-counting dimension obtained by Falconer in [F1] (Theorem 5.3), we have

$$
\begin{aligned}
D_{0}(A) & =\inf \left\{s: \sum_{r=1}^{\infty} \sum_{\mathbf{q} \in S_{k}^{r}} \phi^{s}\left(T_{\mathbf{q}}\right)<\infty\right\} \\
& =\inf \left\{s: \sum_{r=1}^{\infty} k^{r}\left(\psi^{s}\left(a_{1}, a_{2}\right)\right)^{r}<\infty\right\} .
\end{aligned}
$$

Meanwhile Eq. (32) in this section yields

$$
\begin{aligned}
D_{2}(A) & =\sup \left\{s: \sum_{r=1}^{\infty} \sum_{\mathbf{q} \in S_{k}^{r}} \phi^{s}\left(T_{\mathbf{q}}\right)^{-1} k^{-2 r}<\infty\right\} \\
& =\sup \left\{s: \sum_{r=1}^{\infty} k^{-r}\left(\psi^{s}\left(a_{1}, a_{2}\right)\right)^{-r}<\infty\right\} .
\end{aligned}
$$

Since $\psi^{s}\left(a_{1}, a_{2}\right)$ is a decreasing function of $s$, both $D_{0}(A)$ and $D_{2}(A)$ equal the value of $s$ for which $k \psi^{s}\left(a_{1}, a_{2}\right)=1$.

It remains to be shown that the value of $s$ for which $k \psi^{s}\left(a_{1}, a_{2}\right)=1$ is equal to $D_{L}(\Lambda)-1$. Note that if $k a_{1}<1$, then $k \psi^{s}\left(a_{1}, a_{2}\right)=k a_{1}^{s}=1$ for

$$
s=\frac{\log k}{-\log a_{1}}<1 \text {. }
$$


If $k a_{1} a_{2}<1<k a_{1}$, then $k \psi^{s}\left(a_{1}, a_{2}\right)=k a_{1} a_{2}^{s-1}=1$ for

$$
1<s=1+\frac{\log \left(k a_{1}\right)}{-\log a_{2}}<2 .
$$

In each case we have $s=D_{L}(\Lambda)-1$.

\section{Conclusion}

We have shown that the correlation dimension (as defined in (6)) of the attractor of an iterated function system which corresponds to the skinny baker's map is typically a certain function of the contraction rates. This indicates that the correlation dimension is a fundamental dynamical invariant. With its accessibility from a sufficiently long time series, we believe that the correlation dimension will play an important role in both theory and applications of chaotic dynamical systems.

\section{ACKNOWLEDGMENT}

We thank C. Grebogi and H. Weiss for their input. This research was supported by the National Science Foundation (Divisions of Mathematical and Physical Sciences) and by the U.S. Department of Energy (Scientific Computing Staff, Office of Energy Research).

\section{REFERENCES}

[AY] J. C. Alexander and J. A. Yorke, Fat baker's transformations, Ergod. Th. \& Dynam. Sys. 4 (1984), 1-23. MR 86c:58090

[BR] R. Bowen and D. Ruelle, The ergodic theory of Axiom-A flows, Invent. Math. 29 (1975), 181-202. MR 52:1786

[DGOSY] M. Ding, C. Grebogi, E. Ott, T. Sauer, and J. A. Yorke, Estimating correlation dimension from a chaotic time series: when does plateau onset occur? Physica D 69 (1993), 404-424. MR 94g:58136

[F1] K. J. Falconer, The Hausdorff dimension of self-affine fractals, Math. Proc. Camb. Phil. Soc. 103 (1988), 339-350. MR 89h:28010

[F2] K. J. Falconer, Fractal Geometry, Mathematical Foundations and Applications, John Wiley \& Sons, 1990. MR 92j:28008

[F3] K. J. Falconer, The dimension of self-affine fractals II, Math. Proc. Camb. Phil. Soc. 111 (1992), 169-179. MR 92m:28010

[FOY] J. D. Farmer, E. Ott, and J. A. Yorke, The dimension of chaotic attractors, Physica D 7 (1983), 153-180. MR 84m:58022

[G] P. Grassberger, Generalized dimensions of strange attractors, Physics Letters A 97 (1983) 227. MR 84i:58075

[GH] J. S. Geronimo and D. P. Hardin, An exact formula for the measure dimensions associated with a class of piecewise linear maps, Constructive Approximation 5 (1989), 89-98. MR 90d:58076

[GP] P. Grassberger and I. Procaccia, Characterization of strange attractors, Phys. Rev. Lett. 50 (1983) 346; Measuring the strangeness of strange attractors, Physica D 9 (1983) 189. MR 85i:58071

[HP] H. G. E. Hentschel and I. Procaccia, The infinite number of generalized dimensions of fractals and strange attractors, Physica D 8 (1983), 435-444. MR 85a:58064

[KY] J. L. Kaplan and J. A. Yorke, Chaotic behavior of multidimensional difference equations, in Functional Differential Equations and Approximations of Fixed Points, edited by H.-O. Peitgen and H.-O. Walter, Lecture Notes in Mathematics 730, Springer, Berlin, p.204. MR 80k:58074

[P] Y. B. Pesin, On rigorous mathematical definitions of correlation dimension and generalized spectrum for dimensions, Journal of Statistical Physics 71 (1993), 529-547. MR 94d:28008 
[PW] Y. Pesin and H. Weiss, On the dimension of a general class of deterministic and random Cantor-like sets in $\mathbf{R}^{n}$, symbolic dynamics, and the Eckmann-Ruelle conjecture, to appear in Comm. Math. Physics.

[PoW] M. Pollicott and H. Weiss, The dimensions of some self-affine limit sets in the plane and hyperbolic sets, Journal of Statistical Physics 77 (1994), 841-866. MR 95h:58083

[R] A. Renyi, Probability Theory, North-Holland, Amsterdam, 1970. MR 47:4296

$[\mathrm{Ru}] \quad$ D. Ruelle, A measure associated with Axiom A attractors, Amer. J. Math. 98 (1976), 619-654. MR 54:8732

[S1] K. Simon, Hausdorff dimension for non-invertible maps, Ergod. Th. \& Dynam. Sys. 13 (1993), 199-212. MR 94c:58146

[S2] Overlapping cylinders: the size of dynamically defined Cantor-set, in Ergodic Theory of $\mathbf{Z}^{d}$ actions, London Math. Soc. Lecture Notes 228, Cambridge Univ. Press, 1996.

[SY] T. D. Sauer and J. A. Yorke, Are the dimensions of a set and its image equal under typical smooth functions? to appear in Ergod. Th. \& Dynam. Sys.

[Y] L. S. Young, Dimension, entropy and Lyapunov exponents, Ergod. Th. \& Dynam. Sys. 2 (1982), 109-124. MR 84h:58087

Department of Mathematics, Colorado State University, Fort Collins, Colorado 80523 (On leave at: Institute for Mathematics and Its Applications, University of Minnesota, Minneapolis, Minnesota 55455)

E-mail address: chin@ima.umn.edu

Department of Mathematics and Institute for Physical Science and Technology, University of Maryland, College Park, Maryland 20742

E-mail address: bhunt@ipst.umd.edu

E-mail address: yorke@ipst.umd.edu 